\title{
Filigrane
}

Écoutes psychanalytiques

\section{La caverne de Théo. Dans quel espace vivons-nous aujourd'hui ?}

\section{Martin Gauthier}

Volume 28, numéro 2, 2019

URI : https://id.erudit.org/iderudit/1069698ar

DOI : https://doi.org/10.7202/1069698ar

Aller au sommaire du numéro

\section{Éditeur(s)}

Santé mentale et société

ISSN

1192-1412 (imprimé)

1911-4656 (numérique)

Découvrir la revue

Citer cet article

Gauthier, M. (2019). La caverne de Théo. Dans quel espace vivons-nous aujourd'hui ? Filigrane, 28(2), 153-168. https://doi.org/10.7202/1069698ar

\section{Résumé de l'article}

Le progrès technique transforme l'espace et les relations humaines. La physique moderne et la psychanalyse ont souligné les fondements relationnels des espaces dans lesquels nous vivons. Si notre expérience sensible de tous les jours nous place devant un double espace, celui du dedans et celui du dehors, l'articulation entre ces deux espaces a acquis une nouvelle dimension avec les travaux de Winnicott et de Green. À la lumière de l'analyse de Théo, pour qui le cadre analytique apparaissait comme une caverne dangereuse, et de celle du mythe de Persée face à la Méduse, tel que lu par Pasche, l'importance d'un bouclier personnel est discutée. Dans le passage d'un rapport tri-dimensionnel à un rapport bi-dimensionnel, la médiation qu'offre la technologie numérique est ainsi comparée à un bouclier de Persée d'un autre ordre. Son impact est questionné à l'aune de situations cliniques avec les adolescents et de la recherche portant sur le développement des enfants. 


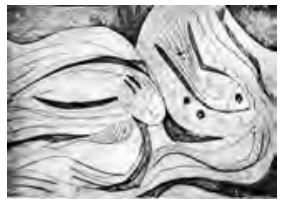

\title{
La caverne de Théo. Dans quel espace vivons-nous aujourd'hui ${ }^{1}$ ?
}

\author{
Martin Gauthier
}

Résumé: Le progrès technique transforme l'espace et les relations humaines. La physique moderne et la psychanalyse ont souligné les fondements relationnels des espaces dans lesquels nous vivons. Si notre expérience sensible de tous les jours nous place devant un double espace, celui du dedans et celui du dehors, l'articulation entre ces deux espaces a acquis une nouvelle dimension avec les travaux de Winnicott et de Green. À la lumière de l'analyse de Théo, pour qui le cadre analytique apparaissait comme une caverne dangereuse, et de celle du mythe de Persée face à la Méduse, tel que lu par Pasche, l'importance d'un bouclier personnel est discutée. Dans le passage d'un rapport tri-dimensionnel à un rapport bi-dimensionnel, la médiation qu'offre la technologie numérique est ainsi comparée à un bouclier de Persée d'un autre ordre. Son impact est questionné à l'aune de situations cliniques avec les adolescents et de la recherche portant sur le développement des enfants.

Mots clés: psychanalyse; espace transitionnel; technologie numérique; troisième topique.

\begin{abstract}
Technical progress changes space and human relationships. Modern physics and psychoanalysis have emphasized the relational basis of the spaces we live in. While our day to day sensible experience places us in front of a double space, the one outside and the one inside each of us, the articulation between the two has taken a new dimension with Winnicott's and Green's work. Using the analysis of Theo, who experienced the analytic setting as a dangerous cavern, and the myth of Perseus, as read by Pasche, the importance of a personal shield is discussed. With its passage from a three-dimensional to a two-dimensional relationship, the mediation offered by digital technology is compared to a Perseus' shield of a different order. Its impact is questioned at the light of developmental research and of clinical experience with adolescents.
\end{abstract}

Key words: psychoanalysis; transitional space; digital technology; third topic.

Le problème n'est pas d'inventer l'espace, encore moins de le réinventer [...], mais de l'interroger, ou, plus simplement encore, de le lire; car ce que nous appelons quotidienneté n'est pas évidence, mais opacité: une forme de cécité, une manière d'anesthésie. (Georges Perec, Espèces d'espaces) 
a faucille a une longue histoire qui remonte avant même la naissance de l'agriculture et le début de l'âge des métaux (Vernus, 2005). L'invention de la faux, qui prit la forme que nous lui connaissons aux XII ${ }^{\mathrm{e}}$ et XIII ${ }^{\mathrm{e}}$ siècles, ne constitua pas un simple progrès technique. Elle signa une petite révolution sociale et suscita maintes résistances avant de se généraliser. Exigeant plus de force, la faux disqualifiait les femmes, jusque-là très présentes lors des moissons. Elle permettait aussi de couper les tiges plus basses et bafouait ainsi un ancien droit d'usage, le droit de chaumage, le chaume étant la paille restant au sol que les plus pauvres pouvaient récolter après la première coupe. Le meilleur rendement de la faux réduisait enfin les besoins en maind'œuvre et le temps nécessaire à la moisson, créant chômage et insatisfaction chez les saisonniers. La faux transforma ainsi les sociétés qui l'adoptèrent. Elle ne modifia pas seulement la manière de travailler; elle bouleversa les rapports avec le territoire et les relations sociales. Ce n'est qu'au XIX $x^{e}$ siècle qu'elle triompha de manière plus générale, mais elle fut vite remplacée par les faucheuses à traction animale qui la reléguèrent à un rôle plus marginal. Les machines prirent ensuite le devant de la scène.

De la faucille à la faux et à la machine, le progrès technique modifie l'espace et les rapports humains. Notre époque numérique avance dans cette foulée et suscite plusieurs questions, en particulier face à l'ouverture illimitée de l'espace virtuel que permet aujourd'hui la technologie mobile et omniprésente. Nous construisons le village global annoncé par McLuhan, mais que savons-nous vraiment de ce village et comment y vit-on? Nous assistons à une délocalisation sans précédent dans l'organisation de la vie sociale, notamment dans l'organisation du travail et du commerce. La délocalisation s'associe moins à une re-localisation définie qu’à une dématérialisation de l'espace de rencontre. La dématérialisation favorise à son tour la fragmentation de l'espace matériel partagé, chacun ${ }^{2}$ pouvant se plonger dans son écran individuel et rejoindre un nouvel espace virtuel. Jamais autant de photographies et autres traces n'auront été accumulées, jamais autant de communications n'auront été échangées, mais connaissons-nous finalement mieux l'espace dans lequel nous vivons et ceux avec qui nous partageons cet espace? Quel impact a notre nouveau rapport à l'espace sur nos relations avec les autres et avec nous-mêmes?

Le psychanalyste d'aujourd'hui est un héritier de l'époque du chemin de $\mathrm{fer}^{3}$ et de la seconde révolution industrielle. Ses outils furent façonnés, au rythme du train à charbon, pour travailler le paysage psychique en le comparant à celui qui défile à la fenêtre d'un wagon en mouvement. De 
nos jours, avec la révolution cybernétique, dans nos trains (de vie) à grande vitesse, rares sont ceux et celles qui ne sont pas plongés dans un écran pour scruter la réalité virtuelle qui y défile. Notre monde est envahi d'images et se découvre en observant la réalité à travers elles. Nous menons aussi des psychanalyses à travers la fenêtre d'un écran; c'est tellement plus pratique pour sauver du temps et franchir les distances en un clic. Nous parlons de «réalité augmentée » quand des apports virtuels se greffent à la réalité matérielle, mais comment bénéficient l'expérience de soi et celle des autres, la subjectivité personnelle et l'intersubjectivité, de toutes ces avancées? La réalité augmentée nous promet-elle des rencontres plus pleines et profondes? Au contraire, sommes-nous invités à vivre dans des mondes parallèles et segmentés? Quel monde fabriquons-nous et comment l'habitons-nous?

\section{Le dehors et le dedans}

La subjectivité demeure encore et toujours un espace mystérieux. Même avec les instruments les plus sophistiqués, à l'heure de tous les espoirs fondés sur les neurosciences, le passage de l'organisation physico-chimique des réseaux neuronaux à la conscience reste une énigme. La conscience émerge, comme l'électricité, mais ses conditions d'émergence nous échappent encore. Notre subjectivité dépend de cette conscience qui fonde notre rapport à la réalité autour des deux vertex que sont le temps et l'espace.

$\mathrm{Du}$ côté spatial, l'expérience sensible nous place devant un espace double, celui du dehors et celui du dedans. À chacun des deux pôles, l'espace se présente comme une étendue contenue dans des limites floues au sein de laquelle se meuvent les objets. Les rapports entre les pôles sont aussi ambigus: est-ce le dedans qui contient le dehors ou le dehors qui contient le dedans? Et quelle est la limite ainsi postulée entre dedans et dehors, comment se trace-t-elle? Dans quel espace vivons-nous?

L'espace n'est pas une évidence, ni pour le physicien ni pour le psychanalyste. Le physicien nous enjoindra de renoncer à un espace absolu et il soulignera le rôle du champ gravitationnel en tant que trame dans laquelle temps et espace évoluent. La disjonction habituelle entre contenant et contenu, entre l'espace et la matière qui s'y meut, cède maintenant la place à une approche unificatrice où temps et espace deviennent eux-mêmes des objets d'étude évoluant comme les autres objets. Il est intéressant de constater cette même révolution pour le psychanalyste qui fait aujourd'hui des processus et de leur genèse les objets de son étude, ne s'attardant plus aux seuls contenus et à leur signification. Le psychanalyste reconnaît aussi le rôle 
des forces d'attraction dans la construction spatiale. L'abord de la maladie schizoïde et le traitement des personnalités qualifiées de borderline ou limite ont transformé la conception des frontières, des espaces et des échanges. Avec leurs modèles et leurs outils, avec le défi de corroborer leurs avancées, physiciens et psychanalystes explorent les conditions qui sous-tendent notre expérience de l'espace.

Tout le travail du psychanalyste concerne l'espace ambigu entre la perception et la représentation. Leur articulation a été une préoccupation de Freud depuis les débuts. En proposant une épreuve de réalité (voir Leclaire et Scarfone, 2000), il soulignait combien les trois termes de cette équation - la représentation, la perception et leur articulation - sont le résultat d'un travail de construction qui caractérise la vie psychique. Celle-ci révèle ainsi la manière qu'a chacun d'articuler perception et représentation, de faire épreuve de réalité. Cette épreuve de réalité concerne tout autant la réalité du dehors que celle du dedans: l'humain est plongé dans la tâche de les réaliser, de leur donner pleine et entière réalité.

Freud a proposé des modèles théoriques pour figurer l'espace du dedans et son organisation en tant qu'appareil effectuant un travail psychique. Il s'y est d'abord aventuré par la porte nocturne de l'espace du rêve et parla déjà en 1900 d'un «travail du rêve» (Freud, 1900). La topographie proposée a évolué, découpant des lieux différents, mais surtout des propriétés différentes associées à ces lieux. Chez Freud, tout espace détermine aussi les qualités dynamiques et économiques des objets qui s'y meuvent. Si la recherche en neurosciences met aujourd'hui l'accent sur les réseaux neuronaux au détriment de localisations uniques - comme en génétique d'ailleurs -, ce fut déjà la première intuition de Freud (1895) pour concevoir le fonctionnement de l'appareil psychique: un réseau de neurones, le moi, exerce une fonction inhibitrice pour réguler l'excitation interne en fonction des conditions externes qui prévalent. L'inventeur de la psychanalyse soulignait qu'une construction était à l'œuvre intérieurement, marquée par les étapes de la maturation biologique et par les événements.

Malgré ses riches incursions dans le champ social, Freud s'est surtout intéressé à l'espace interne et aux forces qui s'y déploient. L'articulation dedans/dehors laissait alors dans l'ombre les premières assises relationnelles de ces forces et de leur organisation. Ses successeurs, à commencer par Ferenczi, ont souligné le rôle fondateur des objets externes (ce que j'appelais plus tôt les «forces d'attraction») dans la constitution des espaces. Dans la foulée du psychanalyste hongrois, nous devons à Winnicott d'avoir non 
seulement insisté sur l'espace relationnel mère-enfant comme base de toute construction spatiale ultérieure, mais aussi d'avoir éclairé les propriétés d'un véritable troisième espace jusque-là négligé, celui de l'articulation entre dedans et dehors, entre représentation et perception. C'est à ce troisième espace qu'il attribua la dénomination d'espace potentiel ou transitionnel lorsque les objets qui s'y meuvent gardent une réalité paradoxale, ni interne, ni externe. Plus encore, il donna une fonction capitale à cette relativité transitionnelle car elle est gage de la créativité personnelle qui seule donne vitalité à la découverte du monde extérieur et aux relations objectales qui s'y nouent. Winnicott (1971) fait ainsi de l'espace transitionnel «le lieu où nous vivons». La vitalité est fruit de l'articulation dedans-dehors quand la transitionnalité prévaut, à défaut de laquelle futilité et soumission s'installent.

Si nous postulions deux espaces au départ, celui du dedans et celui du dehors, nous voici maintenant avec trois espaces interreliés où la clef de voûte de l'édifice est l'articulation processuelle entre dedans et dehors. Cette articulation déplie un espace frontière/transition paradoxal où invention et découverte se mêlent sans nécessité de trancher entre les deux, ce qui constitue la première condition de cette illusion qui sert de tremplin à la vie symbolique. Winnicott insistera aussi sur une seconde condition paradoxale pour que l'aire de jeu prenne toute son ampleur: la réalité externe doit être objectivée, ce qui implique qu'elle survive - on remarque que la question de la vitalité revient encore - à sa destruction. Le psychanalyste britannique soulignait ainsi une loi fondamentale: il n'est d'espace humain qui ne soit relationnel. Le philosophe Merleau-Ponty (1947) soulignait cet aspect au sein même de la perception: «Je ne peux [...] concevoir de lieu perceptible où je ne sois moi-même présent.» La perspective se portait désormais vers les liens, vers les processus aux frontières des espaces, les reliant et les constituant. L'articulation fondant le principe de réalité révélait toute sa complexité.

Depuis, la psychanalyse s'est particulièrement penchée sur les processus menant à la construction de l'espace interne, corrélativement non seulement à l'exploration de l'espace externe, mais aussi particulièrement à la découverte de l'objet externe et du monde intérieur que cet objet comporte aussi. On entre ainsi dans le champ de l'intersubjectivité qui mène aux relations triangulaires. La résolution des conflits rencontrés (EEdipe, phase dépressive) renforce les limites de l'espace personnel tout en favorisant un riche investissement du monde extérieur et une symbolisation ouverte (Laplanche, 1980). Nous pouvons qualifier le champ alors balisé 
de tridimensionnel compte tenu de la triangulation existante (sujet-objetautre de l'objet) et compte tenu de la profondeur de champ inauguré par le monde intérieur de chacun des pôles. À l'opposé, diverses distorsions et stratégies défensives peuvent venir bloquer ou pervertir cette construction. Les mécanismes primitifs tels le déni et le clivage (à l'exemple des rapports fétichistes), ou encore le collage et l'adhésivité (communs dans le spectre de l'autisme), maintiennent des zones plus ou moins grandes de rapports uniou bi-dimensionnels, sans profondeur.

Nous voudrons reprendre cette distinction quant à la dimensionnalité des espaces pour questionner nos relations contemporaines. Auparavant, une situation clinique esquissera l'enjeu spatial des rapports qui s'établissent entre l'individu et son environnement.

\section{Une histoire: la caverne de Théo}

La psychanalyse est une étrange fenêtre sur l'espace du dedans et sur les conditions qui permettent son appropriation. C'est dans l'expérience d'un autre espace, celui physique du bureau comme celui de la rencontre entre analyste et analysant, que l'architecte-analysant peut habiter de plus en plus son propre espace personnel. Pour plusieurs, c'est ainsi qu'ils se découvrent architectes et se donnent le droit de pleinement habiter les lieux. Ce fut le cas pour Théo et sa difficile conquête de l'espace.

Quand Théo vint me consulter, un espace de sa vie parasitait tous les autres: l'espace des toilettes publiques. Depuis le début de l'âge adulte, il lui était impossible d'uriner en présence d'autres personnes; il en perdait l'envie et la capacité. Progressivement, toute sa vie s'organisa autour de l'angoisse suscitée par ce besoin d'uriner dont il anticipait la survenue dans un lieu inopportun. Diverses thérapies avaient donné un nom scientifique à sa phobie - une parurésie -, mais peu de soulagement. La quarantaine bien entamée, il souhaita s'engager dans une psychanalyse, mais dans quel espace allait-il se retrouver?

Aussi concrète et localisée que puisse être une toilette publique, on devine qu'elle constituait un lieu métaphorique pour y figurer quelque chose dont les effets se répercutaient bien au-delà: le retrait de Théo caractérisait toute sa vie. Il vivait seul et centrait ses jours autour d'un travail mal valorisé au sein d'une entreprise caritative. Il fréquentait une femme dans une ville voisine, mais sans intimité sexuelle avec elle depuis longtemps. Sa famille habitait loin et il passait ses longues heures de temps libre dans un atelier de vitrail où il s'appliquait à reproduire des œuvres de maîtres. Dans 
l'ensemble, il suivait scrupuleusement une routine rassurante où les séances d'analyse trouvèrent vite leur place.

En début d'analyse, il n'est pas rare qu'ourdisse une angoisse diffuse suscitée par ce qui risque d'émerger des tréfonds de l'être dans la nouvelle aventure qui s'engage. L'analysant semble alors naviguer à vue, aux aguets, craignant un danger mal défini qu'un trop grand laisser-aller va nécessairement convoquer. La contrepartie fraie également son chemin: l'angoisse que sécrète la perspective de ne jamais parvenir à exprimer ce qui compte vraiment, à ne pas parvenir à l'ouverture nécessaire, pour in fine rester isolé et ignoré. Winnicott (1963) a abordé cet enjeu sous l'angle du conflit entre communiquer et ne pas communiquer. Il y décrivait la saine protestation contre «le fantasme angoissant d'être exploité à l'infini», ce qui équivaut, disait-il, à être mangé ou avalé. La forme plus élaborée du conflit peut s'organiser comme un jeu de cache-cache dans lequel «se cacher est un plaisir, mais n'être pas trouvé est une catastrophe», pour autant que cet «être trouvé» se fasse dans le respect de la part silencieuse du soi, part qui ne communique pas ou qui communique en ses propres termes, à son propre rythme. Chacun des pôles de ce conflit avait son importance pour Théo, qui donnait l'impression de goûter la liberté de parole qui lui était offerte, mais qui avançait avec grandes précautions. L'injonction de bien faire guidait chacun de ses pas.

Je sentais qu'il fallait me faire discret, mais il me fallut du temps avant de reconnaître l'ogre que j'étais pour Théo. Je tardai à saisir toute l'ampleur de la sourde vigilance qui donnait d'abord une touche d'intensité à nos rencontres. Une forme de docilité prévalait, dans un climat qui néanmoins semblait celui d'un investissement positif de l'analyse et de l'analyste. La suite allait démontrer combien la rencontre entre nous était périlleuse. Des signes le suggéraient dès le départ, mais c'est davantage dans leur rigidité tenace que leur envergure se révéla. La position spatiale sur le divan fut un premier indice: Théo peinait à occuper pleinement son espace sans porter le regard vers moi, sans vérifier ma propre position. Il terminait les séances le corps en diagonale, les yeux vers moi. L'enveloppe de rire et d'autodérision qui habillait ses propos et qui balayait rapidement les autres expressions affectives fut un autre indice. Théo s'abandonnait bien peu dans l'espace analytique offert.

Au fil du temps, il apparut que cet espace analytique avait pris la forme d'une dangereuse caverne où régnait la terreur d'être mangé. Quand nous pûmes y donner des formes verbales dans un début de jeu entre nous, Théo 
évoqua la rencontre d'Ulysse et du Cyclope. Que faire quand le retour vers son Ithaque personnelle, vers soi, implique la confrontation au Cyclope? Que faire sinon user de ruse: se travestir en mouton inoffensif et prétendre être oûtis, c'est-à-dire personne? Le combat de personne contre l'ogre: les images d'Homère ouvraient un jeu que la terreur refermait. Et si je parle maintenant de jeu, comme celui de cache-cache, c'est que nous fûmes longtemps dans des lieux où il n'était pas question d'être découvert.

Dans la caverne analytique, Théo restait ainsi replié. S'il s'aventurait hors de son abri, il n'existait pour moi que comme «objet de (votre) désir» et risquait d'être avalé; ou encore, il devenait lui-même l'ogre et je disparaissais. Outre ceux de dévoration, les scénarios d'attaques anales ou castratrices restèrent longtemps empreints d'une terreur radicale qui rendait impossible la rencontre de deux sujets ayant leur espace propre. En outre, toute dépendance était intolérable et vite inversée. Cette inversion faisait alors de Théo le gardien de la caverne, responsable de préserver les lieux des menaces qui guettaient.

Sans détailler l'histoire relationnelle que Théo et moi dépliâmes sur plusieurs années, vous aurez compris que des traumatismes étaient au rendezvous, notamment une séduction homosexuelle par un oncle qui semblait être au début de l'âge adulte, séduction à la suite de laquelle la parurésie débuta. Les propos de l'analysant reprenaient de façon répétitive un petit nombre de scènes d'intrusion, plus ou moins reconnues comme telles, remontant à la petite enfance, autant de tentatives d'emprise par une figure envahissante. Face au danger, laissé à lui-même, Théo était chaque fois un héros solitaire, maquillant sa détresse pour se donner des airs de triomphe. Il était l'élu, au prix d'une dépossession essentielle, sans autre solution que celle de se faire oûtis, personne, tout en cachant son subterfuge.

Ce n'est que lentement, au rythme fiable de nos rencontres et en éclairant les conflits entre nous, que Théo put construire un espace où nous pouvions nous côtoyer sans nous détruire l'un l'autre, où chacun pouvait avoir son propre espace psychique et sa propre puissance, créatrice et relative. Les différentes intimités, à l'exemple des toilettes, perdirent leur charge absolue, meurtrière et castratrice. Pertes, colères et déceptions prirent du relief. Théo compara son travail de deuil et de construction au mouvement de descendre de l'arbre où il avait fait son abri, et ce pour habiter avec les autres une maison dotée d'une porte d'entrée qu'il pouvait faire respecter. Au terme de son analyse, il proposa aussi cette image: "J'étais comme une chaise inoccupée sur la scène d'un théâtre.» 


\section{Méduse et la nécessité d'un bouclier}

Dans le mythe de Méduse sur lequel Freud (1922) s'est penché, Persée tranche la tête de la Gorgone sans croiser son regard sidérant grâce au bouclier poli que lui a donné Athéna. Il évite ainsi d'être transformé en pierre. Pour Freud, Méduse représente le sexe féminin et la menace de castration que sa vue suscite. Dans sa lecture ultérieure, Francis Pasche (1971) a souligné un aspect plus primitif qui nous intéressera particulièrement. Il note que c'est en privant Méduse de la troisième dimension, celle de la profondeur, que Persée peut la vaincre. La Gorgone se voit mise à plat dans le miroir malgré l'illusion de la perspective. Réduite à sa seule image en deux dimensions sur la surface réfléchissante impénétrable, Méduse n’est alors plus un danger. Persée aménage l'espace en limitant Méduse à une image, ce qui procure au héros une marge de liberté pour l'affronter. Méduse perd ainsi son terrible pouvoir de sidération.

Pasche constate l'absence d'une telle liberté chez le psychotique qui n'a pas établi de bouclier protecteur entre le dehors et le dedans. Tout l'envahit, tout l'avale et le persécute. Pour Freud qui propose une interprétation symbolique du mythe, la symbolisation est déjà acquise et permet un traitement de l'angoisse à un niveau plus évolué, celui de la castration. Le psychotique, ou encore chacun de nous dans nos fondements premiers, n'a pas la limite protectrice que l'acquisition de la symbolisation sous-tend. Au niveau plus primitif, nous sommes heurtés frontalement, sans espace personnel protégé. Sans bouclier, il y a incorporation en bloc de la réalité, vécue comme un envahissement par cette réalité sans pouvoir la réduire à une perception et une représentation. Sans bouclier, l'espace est illimité.

Théo n'était pas psychotique et il pouvait ériger, tant bien que mal, un rempart entre lui et moi. Mais il n'avait pas un véritable bouclier protecteur tant les intrusions avaient été importantes sur son parcours. Une distance concrète ou encore un repli s'avéraient toujours nécessaires pour éviter un contact direct qui ne pouvait que mener au pire. Il était à l'étroit, assiégé, en danger d'être assujetti ou subjugué. Contrairement au psychotique, il avait une ébauche de bouclier, mais il ne pouvait se permettre de le déployer et de l'utiliser sans crainte catastrophique. Il restait ainsi clivé et immobilisé entre sa petitesse et sa grandiosité, entre son impuissance et son pouvoir dévastateur. La remise en mouvement impliqua de mieux constituer cette frontière protectrice, soit une limite personnelle différenciant le dehors et le dedans, permettant un jeu entre son espace propre et le mien. 
Ce bouclier de Persée dont Pasche a souligné l'importance trouve un prolongement dans la préoccupation de différents auteurs psychanalytiques pour un troisième modèle topique ${ }^{4}$, modèle qui se préoccupe avant tout de la limite dedans/dehors et des fonctionnements en extériorité. Ceux-ci signent un développement de l'appareil psychique qui n'a pu atteindre le statut d'une unité autonome, avec un bouclier pleinement constitué. André Green (1982) a souligné la dualité de ce bouclier qui inscrit corrélativement une limite entre le dedans et de dehors, de même qu'entre l'inconscient refoulé et le moi. Frontière personnelle et refoulement participent du même fonctionnement d'un sujet unifié qui garde son unité face aux deux pôles que constituent le monde extérieur et le monde intérieur refoulé.

J'insistais plus tôt sur le rôle de l'articulation entre perception et représentation, entre les espaces du dehors et du dedans. Avec Winnicott, cette articulation est devenue un troisième espace, capital pour le développement des deux autres et leur inter-relation. Green décrit une autre caractéristique de cette articulation en mettant en relief la dualité de cette frontière, un feuillet (la limite dedans/dehors) tourné du côté intersubjectif et l'autre feuillet tourné vers l'intrapsychique (le refoulement). Le bouclier que l'ensemble constitue permet d'unir et de séparer. Il protège à la fois contre les affres d'une rencontre sidérante et contre un isolement intolérable. Dans le mythe, cette protection est figurée par le passage d'une rencontre tri-dimensionnelle à une rencontre réfléchie bi-dimensionnelle. Le héros moderne est-il celui capable de composer avec la tri-dimensionalité?

\section{Conquérir l'espace}

En avril 1961, lorsque partit dans l'espace le premier astronaute, le Russe Gagarine, on demanda au philosophe Michel Serres ce qu'il pensait de cette nouveauté. Celui-ci étonna ses interlocuteurs en répondant: "Le nouveau, c'est qu'il ne soit jamais parti!» Dans cette entrevue rediffusée aux Chemins de la philosophie à France Culture le 21 septembre 2018, Serres comparait Gagarine aux navigateurs et aux aventuriers antérieurs dont on pouvait rester longtemps sans nouvelles, alors que l'astronaute n'avait jamais perdu contact avec ceux au sol. Bien avant Internet, déjà l'espace n'était plus le même. Le philosophe postulait que l'espace avait été annulé, ce qui ouvrait, selon lui, une nouvelle approche du temps, de l'espace et des relations humaines. Depuis, le phénomène s'est accru et les communications n'ont jamais été aussi nombreuses et omniprésentes. 
Astronaute ou non, les nouvelles technologies ont conquis notre quotidien et transformé son organisation. Elles favorisent les communications et l'échange d'information comme jamais auparavant. Elles ont permis des avancées techniques dont bénéficient tous les champs humains, tant scientifiques que culturels ou artistiques. En elle-même, la technologie est neutre, l'homme et la femme décident de l'usage qu'ils en font. Le numérique s'inscrit dans une dynamique sociale complexe où il participe aux changements, mais où il ne saurait être isolé ou être tenu seul responsable d'une évolution multi-déterminée. En ce sens, la question spatiale qui anime mon propos a une résonance vaste et multi-factorielle. Il y est important de ne pas perdre de vue la complexité de l'ensemble au moment où je souhaite attirer l'attention sur une modalité particulière ${ }^{5}$.

L'enjeu que j'essaie d'esquisser concerne un aspect du contenant, une caractéristique de l'interface technique qui médiatise notre rapport aux autres en cette ère numérique. En deçà du contenu échangé ou encore du rôle devenu si dominant du médium que constitue l'image dans la culture occidentale actuelle, c'est une dimension de l'image virtuelle, ou plutôt son manque de dimension, que je souhaite mettre en relief. Ramenée à son pôle virtuel, aussi riche en contenu soit-il, la réalité se trouve traduite par la médiation technique en une image réfléchie d'elle-même. L'interface est une sorte de bouclier poli d'un autre ordre que celui de Persée. Aux interactions vives - de vivo, dans un même lieu partagé - se substituent des communications utilisant des images en deux dimensions, sans l'apport des autres modalités sensorielles, sinon le son. La troisième dimension, celle de la profondeur ou de l'épaisseur, celle sagittale du champ, celle de la perspective, perd en importance. Ce glissement est subtil, mais la qualité de l'expérience relationnelle est alors différente.

La piste du tri-dimensionnel nous oriente vers nos connaissances du développement de l'enfant. On y apprend que seule la troisième dimension de la profondeur permet de concevoir un espace à partir d'une perception visuelle (ou sensorielle en général). À cet égard, les différentes modalités sensorielles sont nécessaires à la construction d'une réalité plus complexe et pluridimensionnelle, ce qui est impossible avec la seule représentation visuelle. Les études précoces ont ainsi démontré l'importance des transferts entre les modalités sensorielles pour la construction des représentations mentales, tandis que les études longitudinales ont permis de constater les acquisitions progressives faites par les enfants dans leur appréhension de l'espace matériel, jusqu'à l'étape des opérations formelles à l'adolescence 
(Douriez-Pinol, 1974). Du côté de la pathologie, les difficultés que présentent les autistes mettent aussi en relief l'importance des transferts transmodaux. La dyssensorialité, soit l'échec de la synchronisation polysensorielle, est aujourd'hui une théorie physiopathologique de l'autisme qui trouve un soutien significatif tant du côté des recherches cognitives que du côté de la clinique psychanalytique (Golse, 2017; Guinot et Golse, 2018). Sachant que la co-modalisation est un agent central de l'accès à l'intersubjectivité, ce déficit éclaire les comportements autistiques d'agrippement et de collage, signes de rapports demeurant uni- ou bi-dimensionnels avec l'entourage.

Auprès des enfants et des adolescents, l'expérience clinique démontre le possible mésusage des outils numériques par ceux qui sont déjà les plus vulnérables. L'espace virtuel permet alors une forme de relation qui peut aussi être qualifiée d'uni- ou bi-dimensionnelle, particulièrement en collage ou dans un rapport addictif et de grand contrôle, protégeant l'individu contre les angoisses suscitées par la rencontre de chair et d'os en trois dimensions. L'outil lui-même n'est pas la cause de l'usage qui en est fait, mais ses propriétés bi-dimensionnelles peuvent servir de bouclier externe à ceux qui n'ont pas pleinement le leur en eux. Dans les cas plus graves, le monde virtuel prend les qualités d'un monde imaginaire coupé de la réalité extérieure. Certains adolescents avalent le monde par la bouche de leur écran et sont avalés par cet écran, au détriment de toute autre relation significative. Et même au quotidien plus ordinaire, que dire des angoisses de séparation si vite suscitées aujourd'hui quand la communication attendue fait défaut, quand le contact est soudainement rompu, trahissant un rapport différent à l'espace?

Questionner la bidimensionnalité numérique implique une interrogation de toute l'expérience relationnelle, soit celle d'union et de séparation, ce que comporte l'enjeu de la double limite. Ce que je cherche à mettre en relief concerne un possible glissement depuis l'image concrète bi-dimensionnelle vers des rapports interpersonnels que l'on peut aussi qualifier de bi-dimensionnels. Dans notre culture numérique où le nombre et la richesse des interactions in vivo diminuent, où le virtuel a aussi envahi la petite enfance, ne peut-on pas appréhender un subtil mouvement d'aplanissement, de mise en surface, qui transforme le rapport que nous entretenons avec l'espace du dedans et avec celui des autres? Les forces favorisant l'investissement du pôle virtuel sont puissantes, le contenu offert est stimulant, mais il est aussi plus distancié, sans profondeur de champ, tout en étant sous un plus grand contrôle par l'individu qu'autrui ne l'est. Si les interactions in vivo ont un 
rôle capital pour le développement de l'espace symbolique personnel et de l'intersubjectivité, quelle influence aura la multiplication des interactions passant par la technique? Quel rôle aura la technologie et sa médiation externe dans le fonctionnement du bouclier personnel de chacun?

La modalité du contenant bi-dimensionnel n'est qu'un aspect du tableau. Si on ajoute l'ampleur des communications médiatisées par la technologie, on constate combien celle-ci alimente l'espace symbolique et imaginaire d'une manière inédite, réduisant d'autant l'espace imaginatif né du silence et de la seule stimulation intérieure. De plus, les images extérieures envahissent toutes les sphères de la vie, jusque dans l'intimité, alors que les géants commerciaux (les nouveaux ogres) cherchent à faire tomber les barrières de la confidentialité et à étendre leur contrôle sur la sphère privée. De leur côté, les internautes sont prêts à de grandes concessions en échange d'un accès aux réseaux sociaux. Quelles conséquences cela aura-t-il sur l'espace où nous vivons?

Le numérique permet des avancées extraordinaires, par exemple en rendant disponible des thérapies psychanalytiques à nombre de Chinois pour lesquels une telle expérience était auparavant irréalisable ${ }^{6}$. Viendra peut-être plus rapidement que nous le croyons un temps où les rencontres virtuelles seront considérées préférables partout pour des raisons économiques et pour éviter la pollution engendrée par les déplacements physiques. Cependant, gardons à l'esprit les transformations que la technique introduit subrepticement dans l'espace et dans nos rapports interpersonnels.

\section{Pour conclure: les nouvelles relations}

À l'heure actuelle, la technologie numérique transforme nos rapports au monde et à nous-mêmes, comme la faux l'avait fait en son temps. Elle est omniprésente et ses productions sont infinies. Dans le grand laboratoire de délocalisation que nous expérimentons, quel espace habitons-nous?

La psychanalyse a mis en relief combien (et comment) nous habitons des espaces paradoxaux, tant au dedans qu'au dehors de soi, construits dans et par les relations que nous établissons. Plus encore, c'est le lien entre les espaces qui détermine la vie que nous y menons. Le plein déploiement des espaces interne et externe repose sur des rapports tri-dimensionnels, impliquant la subjectivité de chacun et l'espace partagé. De tels rapports sont possibles pour autant que chacun ait su développer son bouclier personnel, cette double limite transitionnelle chère à Winnicott et Green, déficiente dans le cas de mon patient Théo. 
Derrière l'affirmation d'une annulation de l'espace (Serres), entendons une transformation des frontières et des processus sous-tendant ces frontières. Entendons aussi une transformation de l'expérience de la séparation et de l'absence, tant physique que psychique. Entendons enfin que si l'expérience de la séparation n'est plus la même, celle de l'union change aussi nécessairement. La relation n'est plus ce qu'elle était, et le monde à sa suite. C'est en tout cas ce que j'ai voulu explorer en rappelant certains acquis dans ce développement spatial et en questionnant la médiation bi-dimensionnelle qu'introduit la technologie. Sans glisser vers l'usage pathologique des outils numériques, on peut s'interroger sur la transformation induite par cette médiation. J'interroge un subtil aplanissement, mais je n'ai mis en relief qu'un seul facteur au sein de bien d'autres. L'outil lui-même, surtout lorsqu'il est miniaturisé pour tenir dans la main, nous accompagne partout, avec un impact direct et indirect sur les rapports sociaux. Ce qui circule comme contenu virtuel et la forme prise par ces contenus, de même que les véhicules mêmes de l'échange (les médias sociaux en particulier), transforment également tant les liens sociaux que la manière avec laquelle chacun se présente et se représente à soi et aux autres (Simanowski, 2018). Là aussi le médium transforme non seulement le message, mais aussi toute l'économie personnelle et relationnelle.

Être seul, dans le noir et en silence, est au fondement ontologique de l'être. L'espace s'y présente et s'y re-présente comme une abstraction subjective constituant, avec la dimension du temps, le cadre essentiel de notre condition humaine. Pour l'aborder, la technologie actuelle offre une formidable médiation dont on n'a pas encore exploré tous les horizons. J'ai comparé celle-ci à un bouclier poli d'un autre ordre que celui mythique de Persée, transformant subtilement les conditions dans lesquelles chacun construit son propre bouclier personnel. Déjà des données scientifiques existent quant à l'impact de l'usage de la technologie numérique sur les capacités d'attention et d'orientation, ou encore sur la mémoire (Sparrow, Liu et Wegner, 2011). Le psychanalyste voudra suivre son influence sur les lieux où nous vivons.

Martin Gauthier martin.gauthier3@sympatico.ca 


\section{Notes}

1. Version française remaniée d'une présentation faite au colloque multidisciplinaire organisé par la Société portugaise de psychanalyse sur le thème (In) finite Space: Psychoanalysis and Interception of Places à Lisbonne, en mars 2019.

2. On comprendra que l'usage du masculin, ici «chacun», là «le psychanalyste», a partout valeur épicène dans le texte. La question du genre peut aussi être abordée comme une autre forme de délocalisation, lorsque le corps biologique ne semble pas pouvoir être habité comme tel.

3. Les enjeux économiques et sociaux associés au vaste développement des réseaux ferroviaires en Europe sous-tendaient l'intérêt porté à l'hystérie et au trauma psychique à la fin du XIX ${ }^{\mathrm{e}}$ siècle (Ellenberger, 1968). C'est aussi vers un accident de chemin de fer que Freud se tourna pour aborder le rêve traumatique dans Au-delà du principe de plaisir (1920 a), même si la Grande Guerre lui en donnait certes bien d'autres exemples. La même année, dans Psychogenèse d'un cas d'homosexualité féminine (1920 b), une psychanalyse est explicitement comparée à un voyage en train, avec d'abord les préparatifs, puis le trajet et ses étapes.

4. Une troisième topique est maintenant proposée depuis quelques années (Brusset, 2006; Green, 1993). Elle se veut une perspective de réponse aux difficultés de transformation rencontrées dans l'usage clinique des deux grands modèles antérieurs mis de l'avant par Freud. Elle s'adresse particulièrement aux vicissitudes entourant la fermeture du moi pour mettre en place un espace psychique personnel et différencié, ce qui convoque aussi le problème des conditions d'accès à une conflictualité intra-subjective. Elle offre ainsi un point de vue préalable au travail psychique sous-tendu par la mise en place de la double limite (Green, 1982), limite rendant pleinement opérationnelles les deux topiques freudiennes (Gauthier, 2006; Reid, 2006).

5. Il est toujours périlleux de s'aventurer du côté social à partir d'une expérience clinique surtout glanée dans le travail avec des individus et des familles. Dans une tentative antérieure (Gauthier, 2018), je m'étais servi d'un concept tracé en pointillé chez Winnicott, la défense maniaque de la vie quotidienne, pour aborder l'importance du divertissement dans notre culture actuelle. La question des espaces poursuit cette téméraire réflexion sur nos enjeux collectifs par une autre voie.

6. Mon propos donne un argument métapsychologique à la position adoptée par l'Association psychanalytique internationale concernant l'analyse d'un candidat en formation psychanalytique. Le règlement réserve la psychanalyse à distance pour des circonstances exceptionnelles et exige un certain nombre de séances in vivo. Le principe reconnaît que la richesse de la rencontre en chair et en os est inégalée par la technologie. Cela ne nie surtout pas la possibilité qu'un travail fructueux puisse avoir lieu à travers les outils technologiques, mais il apparaît important de ne pas nier les différences, notamment au niveau des espaces mobilisés.

\section{Références}

Brusset, B. (2006). Métapsychologie des liens et troisième topique. Revue française de psychanalyse, 70 (5), 1213-1282.

Dourez-Pinol, M. (1974). Les fondements de la sémiotique spatiale chez l'enfant. Nouvelle revue de psychanalyse, 9, 171-191.

Ellenberger, H. F. (1968). La conférence de Freud sur l'hystérie masculine (15 octobre 1886). Étude critique. Dans Les mouvements de libération mythique et autres essais sur l'histoire de la psychiatrie (p. 153-178). Montréal: Les Éditions Quinze/Critère, 1978. 
Freud, S. (1895). Esquisse d'une psychologie scientifique. Dans La naissance de la psychanalyse (p. 307-396), Paris: Presses universitaires de France, 1973.

Freud, S. (1900). L'interprétation des rêves. Paris: Presses universitaires de France, 1967.

Freud, S. (1920 a). Au-delà du principe de plaisir. Dans Essais de psychanalyse. Paris: Payot, 1985.

Freud, S. (1920 b). Psychogenèse d'un cas d'homosexualité féminine. Dans Névrose, psychose et perversion (p. 245-270). Paris: Presses universitaires de France, 1973.

Freud, S. (1922). La tête de Méduse. Dans Résultats, idées, problèmes II (p. 49-50). Paris: Presses universitaires de France, 1985.

Gauthier, M. (2006). Troisième topique et psychanalyse d'enfant. Revue française de psychanalyse, 70 (5), 1483-1489.

Gauthier, M. (2018). L'interprétation: pour une remise en chantier. Filigrane, 27 (2), 17-27.

Golse, B. (2017). Détruire ou effacer l'objet. Les mécanismes autistiques et leur impact transférentiel et contre-transférentiel. Journal de la psychanalyse d'enfant, 7 (1), 83-102.

Guinot, M. et Golse, B. (2018). Les destins de la dyssensorialité. Enfant singulier, particularités plurielles. Journal de la psychanalyse d'enfant, 8 (1), 167-184.

Green, A. (1982). La double limite. Dans La folie privée (p. 293-316). Paris: Gallimard.

Green, A. (1993). Le travail du négatif. Paris: Les Éditions de Minuit.

Laplanche, J. (1980). Problématiques II: castration; symbolisations. Paris: Presses universitaires de France.

Leclaire, M. et Scarfone, D. (2000). Vers une conception unitaire de l'épreuve de réalité. Revue française de psychanalyse, 64 (3), 885-912.

Merleau-Ponty, M. (1946). Le primat de la perception et ses conséquences philosophiques. Paris: Éditions Verdier, 2014.

Pasche, F. (1971). Le bouclier de Persée ou psychose et réalité. Revue française de psychanalyse, 35 (5-6), 859-870.

Perec, G. (1974). Espèces d'espaces. Paris: Galilée.

Pontalis, J.-B. (1990). La force d'attraction. Paris: Seuil.

Reid, W. (2006). De l'ensemble individu/environnement à la troisième topique: la pulsion, le narcissisme, l'emprise et la relation d'objet. Revue française de psychanalyse, 70 (5), 1543-1557.

Simanowski, R. (2016). Facebook Society: losing ourselves in sharing ourselves. New York: Columbia University Press.

Sparrow, B., Liu, J. et Wegner, D. M. (2011). Google effects on memory: Cognitive consequences of having information at our fingertips, Science, 333, 776-778.

Vernus, M. (2005). La faux. De l'outil au symbole. Salins-les-Bains: Musées des techniques et cultures comtoises.

Winnicott, D. W. (1963). De la communication et de la non-communication. Dans Processus de maturation chez l'enfant (p. 151-168). Paris: Payot, 1983.

Winnicott D. W. (1971). Jeu et réalité. Paris: Gallimard, 1975. 